\title{
\#Ayotzinapa, origen y evolución del movimiento social mexicano en Twitter
}

\author{
\#Ayotzinapa, origin and evolution of the mexican social \\ movement on Twitter
}

\section{Rocío Abascal Mena'}

Universidad Autónoma Metropolitana - Cuajimalpa, México

mabascal@correo.cua.vam.mx

\section{RESUMEN}

México se consternó una vez más en septiembre de 2014 tras la noticia de los desaparecidos de Ayotzinapa -43 de ellos-, todos de la Escuela Normal Rural del Estado de Guerrero, al sur de la Ciudad de México. Las redes sociales, ya utilizadas en movimientos como \#YoSoy132, fueron testigos del reclamo de los mexicanos. Siendo Twitter la red más utilizada para manifestar el reclamo de los usuarios al Gobierno Mexicano porque los devolvieran vivos. En este artículo, mostramos el resultado del estudio de un amplio corpus de tuits \#Ayotzinapa en torno a la tragedia. Asimismo, estudiamos en particular las etiquetas constitutivas del joven género discursivo, cuyo anonimato, minimalismo y velocidad comunicativa, pueden revelar algunas facetas de las críticas más fuertes hechas al gobierno. Nuestra hipótesis se basa en que a partir de la popularidad en el uso de hashtags asociados hay una evolución de estos con el fin de llegar a una mayor audiencia. Nuestras conclusiones muestran que es necesaria una transformación de las etiquetas, con el paso del tiempo, para permanecer en la red social.

Palabras clave: Redes sociales, Twitter, popularidad, correlación.

\begin{abstract}
Mexico was shocked once again in September 2014 over the missing Ayotzinapa students - 43 of themnews, all from the Rural Normal Guerrero State School in southern Mexico City. Social networks, already in use by movements like \#YoSoy132, witnessed the Mexicans' claim. Twitter was the most popular to express the people's claim to the Mexican government asking to return students alive. In this article, we show the study results of a large corpus composed by \#Ayotzinapa tweets. Moreover, we also especially study, the young discursive genre labels, whose anonymity, minimalism and communicative speed, can reveal some facets of the government's strongest criticisms. Our hypothesis states that from the popular use of the associated hashtags there is an evolution of them in order to reach a wider audience. Our findings show that labels' transformation is required, over time, to remain in the social network.
\end{abstract}

Keywords: Social network, twitter, popularity, correlation.

Historial del artículo:

Recibido: 10 de octubre de 2015. Aprobado: 16 de noviembre de 2015. Disponible en línea: 30 de diciembre de 2015

1 Dra. en Informática e Información para la Sociedad por el Instituto Nacional de Ciencias Aplicadas (INSA) de Lyon, Francia. Profesor-

Investigador de tiempo completo en la Universidad Autónoma Metropolitana - Cuajimalpa, Ciudad de México. 


\section{INTRODUCCIÓN}

Existen, actualmente, nuevas formas de comunicación mediadas por la computadora como lo son las llamadas redes sociales digitales siendo estructuras sociales formadas por nodos -personas, grupos u organizaciones-, que están vinculados respecto a intereses y/o puntos de vista en común. Éstas han propiciado una comunicación distinta a la oral en donde la restricción del tiempo y el espacio generan nuevas prácticas lingüísticas.

Entre las redes sociales digitales se encuentra Twitter que es una red social digital cuya característica principal son sus mensajes que se envían teniendo una longitud máxima de 140 caracteres, llamados tuits. Estos pueden ser almacenados y categorizados en temas a partir del uso de etiquetas (\#hashtags) permitiendo seguir, buscar y encontrar mensajes del mismo tema. Twitter, así como otras redes digitales, permite a los usuarios ser creadores de la información, volverse elementos claves en la discusión de un determinado tema. Por lo tanto, Twitter ya ha sido utilizado, en varias ocasiones, como un medio para la discusión política en contextos locales y nacionales, protestas y movimientos activistas como las ocurridas en México con \#YoSoy132. Twitter se convirtió en un canal legítimo de comunicación en la arena política como un resultado de la campaña presidencial del 2008 realizada en Estados Unidos (1).

Twitter incorpora nuevos elementos tecno-discursivos como lo son el acortamiento de palabras o el uso de etiquetas en un determinado tema. Existen varios ejemplos de investigadores que se han enfocado en el estudio de estos elementos. Entre ellos está Cunha et al. (2) quien estudió la propagación de los hashtags dentro de comunidades de habla, es decir, grupos de personas cuyos miembros lingüísticamente se influencian entre sí. En la investigación se encontró una relación entre la distribución de los hashtags dentro de los rankings de frecuencia y la longitud de las etiquetas para que éstas sean adoptadas por otros usuarios. Por su parte, Romero et al. (3) estudió la manera en que una información se difunde en Twitter a partir del uso del análisis de las variaciones de las características de difusión en distintas temáticas. Para ello, calcularon la probabilidad de adopción de un hashtag basado en el número de exposiciones. Chew \& Eysenbach (4) encontraron la existencia de variabilidad en el uso y contexto de las etiquetas al estudiar, durante un periodo de tiempo, el uso de \#H1N1 y \#swineflu en los tuits generados durante 2009. Los hashtags han sido estudiados para determinar los sentimientos emitidos en todo el tuit. Por ejemplo, Rodríguez et al. (5), a partir de un corpus electoral, categoriza unas diez etiquetas en positivas, negativas o neutrales y hace un conteo de los tuits que las incluyen para tratar de determinar si las etiquetas pueden ser utilizadas con el fin de identificar sentimientos emitidos en un tuit.

Es posible encontrar estudios en los que se habla acerca de las comunidades que son grupos de usuarios organizados con un objetivo en común. Las comunidades están, principalmente, asociadas a un grupo de etiquetas $(6,7)$ permitiendo la formación de públicos ad-hoc alrededor de temas específicos.

Chris Messina propone, en el 2007, el uso del símbolo \# para mejorar la contextualización y el filtrado de Twitter (8). El uso del etiquetado amplía la capacidad del mensaje al convertir a la etiqueta en un identificador para (a) re-documentar el tuit haciendo uso de la hipertextualización, (b) categorizar el tuit dentro de grupos ad-hoc y áreas temáticas, (c) generar comentarios metadiscursivos, (d) crear marcas expresivas, (e) crear interpretaciones personales e (f) identificar de manera genérica una temática. En el caso de la generación de comentarios metadiscursivos se toma la definición de Hyland \& Tse (9) donde explica que son estrategias utilizadas por el escritor para

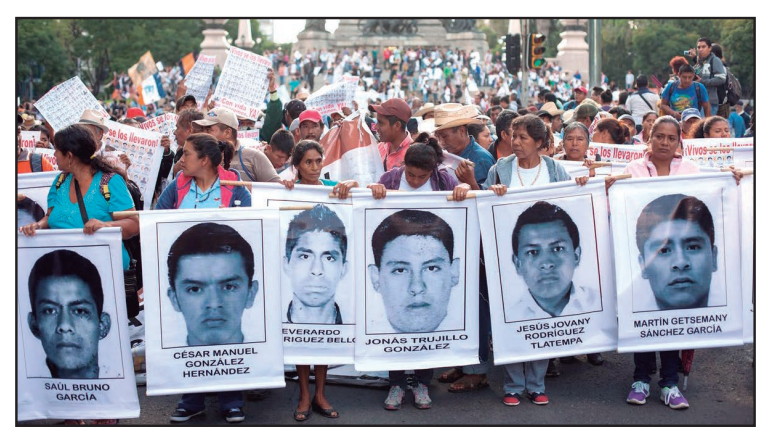

Figura $\mathrm{N}^{\circ}$ 1: Movilización por desaperecidos de Ayotzinapa.

organizar el texto y que permiten conectar distintas ideas. Este es un elemento crucial en la escritura argumentativa al facilitar la persuasión (10).

Rusell (11) y Poell (12) se refieren al uso de procesos metadiscursivos para persuadir a los tuiteros en torno a los movimientos suscitados durante la Primavera Árabe. Rusell (11) afirma que la conciencia y el metadiscurso inspirado en estos tuits tienen sus precedentes: se trata de un fenómeno recurrente en la historia del periodismo profesional, que se origina, particularmente, en momentos de crisis política o de polarización (13). Según Georgakopoulou (14), lo que más destaca en estas nuevas formas de narrativa digital es el potencial para una mayor interactividad con el usuario lector y el abanico de formas de involucramiento del lector, que pueden hasta cambiar decisivamente el curso e interpretación de la historia narrada.

Actualmente, el uso de la etiqueta genérica no se ha dado del todo al encontrar que los usuarios etiquetan 
su tuit con múltiples hashtags para poder llegar a una mayor audiencia. Bruns y Burgess (7) argumentan que el uso de una etiqueta genérica permitiría organizar a los tuits dentro de una gran variedad de categorías haciendo mucho más fácil la desambiguación con tuits que no tienen nada en común.

La desaparición de 43 estudiantes normalistas de Ayotzinapa en septiembre de 2014 en México (figura 1), generó una gran movilización masiva digital a partir de la creación de tuits etiquetados con \#Ayotzinapa, \#TodosSomosAyotzinapa, \#yamecansé - \#justiciaglobalporAyotzinapa. En este artículo se presentan los resultados de un análisis alrededor del uso de los hashtags como elementos básicos en la composición del mensaje. El objetivo es analizar la popularidad de los hashtags, sus relaciones con otros y su evolución a través del tiempo como elementos claves para la difusión de la información. La investigación se realizó a partir de la recuperación de tuits etiquetados con \#Ayotzinapa de septiembre a diciembre 2014.

\section{MATERIAL Y MÉTODOS}

Para la recuperación de los datos se utilizó Twitter con el fin de monitorear una diversidad de hashtags asociados a \#Ayotzinapa. Se utilizó el lenguaje de programación R (https://www.r-project.org/) que contiene una librería llamada twitteR y con la que se puede utilizar el API de esta aplicación y así recuperar los tuits que se generan en el momento. Así, se conformó un corpus para cada una de las etiquetas analizadas. Aunque en este artículo sólo se muestran las etiquetas asociadas a un determinado hashtag, los datos obtenidos también contienen otros parámetros como lo son: nombre del autor principal, fecha, id, si se trata de un reply o no, etc.

El proceso consiste en extraer automáticamente todos los tuits que contienen el hashtag \#Ayotzinapa e ignorar los tuits duplicados (se eligió considerar sólo el primero de acuerdo a la fecha). El texto se escribe con minúsculas (para homogeneizar la variedad de estilos por parte de los autores). En total se recuperaron 350323 tuits en un periodo de septiembre a diciembre de 2014 .

\section{RESULTADOS}

El movimiento social de \#Ayotzinapa generó bastante participación por parte de los mexicanos pero también por ciudadanos de otras partes del mundo. Por esta razón, es posible encontrar una gran variedad de hashtags que se utilizan junto con \#Ayotzinapa para ampliar la difusión de lo ocurrido. Entre los hashtags más utilizados están: \#AyotzinapaSomosTodos,
\#México, \#YameCansé, \#FueElEstado, \#FueEIEjercito, \#EPNBringThemBack, y \#FueraPeñaNieto.

Todos los tuits analizados tienen como principal característica el uso de \#Ayotzinapa independientemente de que se utilicen otros hashtags. Por lo tanto, se puede afirmar que durante todo el movimiento prevalece como estandarte \#Ayotzinapa acompañado de elementos que van ocurriendo, durante el tiempo, y que fortalecen el movimiento haciéndolo más visible y permanente.

Los resultados que a continuación se muestran están basados en dos factores:

1) el Índice de Popularidad (IP)

2) el Grado de Correlación (GC). El IP se mide del 0 al 100 y muestra qué tan popular es el hashtag con respecto a la información de todo Twitter. El hashtag más utilizado en Twitter obtendrá un 100. Por su parte, el GC se mide como el porcentaje de los tuits con el hashtag buscado que también utilizan el relacionado.

Tabla N 1: Popularidad y correlación con \#Ayotzinapa.

\begin{tabular}{lcc}
\hline hashtag & Popularidad & $\begin{array}{c}\text { Correlación con } \\
\text { \#Ayotzinapa }\end{array}$ \\
\hline \#México & 72,6 & $3,9 \%$ \\
\#YaMeCansé2 & 58,9 & $2,7 \%$ \\
\#FueElEstado & 55,7 & $1,8 \%$ \\
\#EPNBringThemBack & 50,6 & $1,7 \%$ \\
\#AyotzinapaSomosTodos & 54,5 & $2,5 \%$ \\
\#YaMeCansé & 61,4 & $5,6 \%$ \\
\hline
\end{tabular}

Cuanto más fuerte sea la correlación, mayor será la relación que tienen dos diferentes hashtags.

\section{\#Ayotzinapa}

Nuestro análisis comienza con la etiqueta \#Ayotzinapa el cual aparece con mayor frecuencia con: 1) \#México, que tiene un IP de 72,6, y 2) \#YameCansé, que tiene un IP de 61,4. En la tabla 1 se muestra el GC de \#Ayotzinapa el cual está asociado con \#México en el $3,9 \%$ de sus tuits y con \#YaMeCansé en el 5,6 \% de los tuits.

Los tres hashtags con mayor popularidad relacionados con \#AyotzinapaSomosTodos son: 1) \#Ayotzinapa, que tiene un IP de 62,9, 2) \#YameCansé, que tiene un IP de 61,4 y 3) \#YaMeCansé2, que tiene un IP de 58,9.

Alanalizarel GC, en la tabla 2, \#AyotzinapaSomosTodos está asociado con \#FueEIEstado en el 11,1 \% de sus tuits, con \#FueraPeñaNieto en el 8,7 \% de sus tuits y con \#Ayotzinapa en el 9,6\% de los tuits. En la tabla 2 se muestra el IP y el GC de \#AyotzinapaSomosTodos con 
hashtags declarativos tales como: \#FueraPeñaNieto, \#FueElEstado o \#DestituciónyJuicioEPN4. Los hashtags asociados muestran un gran descontento con el actual Presidente de México. De igual forma, se encuentra la introducción de \#EPNBringThemBack para alcanzar públicos internacionales.

Algunos ejemplos de tuits con \#Ayotzinapa son:

- AMLO @lopezobrador_es una mala persona ,porque culpa a @epn por \#Ayotzinapa si el peje puso a abarca en iguala era su candidato

- Irreverente, Informado y conciso \#EIPulsoDeLaRepublica \#AYOTZINAPA ¿ENCABEZADO MUNDIAL?

- El papa "eleva sus oraciones" por los estudiantes desaparecidos de \#Ayotzinapa

\section{\#AyotzinapaSomosTodos}

\begin{tabular}{lcc}
$\begin{array}{c}\text { Tabla N 2: Popularidad } \\
\text { \#AyotzinapaSomosTodos. }\end{array}$ & $\begin{array}{c}\text { correlación con } \\
\text { hashtag }\end{array}$ & Popularidad \\
\hline \#FueraPeñaNieto & $\begin{array}{c}\text { Correlación con } \\
\text { \#Ayotzinapa } \\
\text { SomosTodos }\end{array}$ \\
\hline \#FueElEstado & 53,1 & $8,7 \%$ \\
\#Ayotzinapa & 62,7 & $11,1 \%$ \\
\#DestitucionyJuicioEPN4 & 46,5 & $9,6 \%$ \\
\#YaMeCanse2 & 58,9 & $2,8 \%$ \\
\#EPNBringThemBack & 50,6 & $2,5 \%$ \\
\#AyotzinapaNoSeOlvida & 42,9 & $4,2 \%$ \\
\#YaMeCansé & 61,4 & $2,6 \%$ \\
\#FueEIEjercito & 44,2 & $6,5 \%$ \\
\hline
\end{tabular}

\#AyotzinapaSomosTodos invoca un grito de justicia de todos los mexicanos. La desaparición de los 43 estudiantes ha sido el punto de quiebre entre un antes y un después para México. No sólo las instituciones de educación superior, mediante sus jóvenes, piden justicia sino que instituciones como la Iglesia lo hacen a partir también de varias ejecuciones acontecidas hacia sacerdotes. \#AyotzinapaSomosTodos aparece, la gran mayoría de las veces, acompañado de otros hashtags que permiten describir el contexto del movimiento.

Algunos ejemplos de tuits con \#AyotzinapaSomosTodos son:

- Se radicalizan normalistas en el \#lstmo \#Oaxaca por estudiantes desaparecidos de \#Ayotzinapa \#AyotzinapaSomosTodos

- Los familiares de los normalistas desaparecidos de \#Ayotzinapa ya se encuentran en Los Pinos para reunirse con@EPN \#AyotzinapaSomosTodos
- ¿Qué va a cosechar un país que siembra cuerpos? \#Ayotzinapa \#AyotzinapaSomosTodos

\section{\#YaMeCansé}

Durante una conferencia de prensa el Procurador General de la República, Jesús Murillo Karam, lanzó un "Ya me cansé" al dar a conocer una nueva versión del caso de los 43 estudiantes. Su frase se convirtió en un reclamo de la sociedad civil que en cuestión de horas con el hashtag \#YaMeCansé se encontraron frases como: "Si ya te cansaste vete", "Si al procurador ya se le acabó la 'pila', por dignidad debiera retirarse", "Me cansé de la clase política que tiene secuestrado a mi país, de la clase que corrompe, que miente, que asesina, yo también ya me cansé". Este \#YaMeCansé que ha sido reproducido y multiplicado hasta la saciedad, recuerda el grito que recorrió México de norte a sur en 2011 , el "hasta la madre" pronunciado por el activista Javier Sicilia, fundador del Movimiento por la Paz con Justicia y Dignidad, y que ahora tenía

Tabla №3: Popularidad y correlación con \#YaMeCansé.

\begin{tabular}{lcc}
\hline hashtag & Popularidad & $\begin{array}{c}\text { Correlación } \\
\text { con } \\
\text { \#YaMeCansé }\end{array}$ \\
\hline \#AccionGlobalporAyotzinapa & 52,6 & $7,7 \%$ \\
\#Ayotzinapa & 62,9 & $7,1 \%$ \\
\#1DMX & 53,4 & $6,9 \%$ \\
\#20NovMx & 46,1 & $3,1 \%$ \\
\#TodosSomosCompas & 43,5 & $2,8 \%$ \\
\#Articulo39RenunciaEPN & 43,4 & $2,3 \%$ \\
\#YaMeCanse2 & 58,9 & $2,5 \%$ \\
\#AyotzinapaSomosTodos & 54,5 & $2,1 \%$ \\
\#TodosSomosAyotzinapa & 50,8 & $2,0 \%$ \\
\hline
\end{tabular}

nuevas variantes: "Algunos se cansan, otros estamos hasta la madre", decía un 'meme' sobre la foto del procurador.

Algunos ejemplos de tuits con \#YaMeCansé son:

- Well, that's it! I'm moving to MEXICO to get a job on a SOAP OPERA!! I hear they PAY 10 MILLION! \#Mexico \#YaMeCanse

- \#YaMeCanse De que México se haya convertido en una NARCOFOSA gracias a @FelipeCalderon y @ EPN

- No podemos quedarnos atrapados en Ayotzinapa: Peña Nieto \#ComoNoSonSusHijos \#YaMeCansé

Los tres hashtags con mayor popularidad relacionados con \#YaMecansé son: 1) \#Ayotzinapa, que tiene un IP de 62,9, 2) \#YameCansé2 que tiene un IP de 58,9 y 3) \#AyotzinapaSomosTodos con 54,5 de IP. En la tabla 3 
se muestra el GC de \#YaMeCansé que está asociado con \#AccionGlobalporAyotzinapa en el 7,7 \% de sus tuits, con \#Ayotzinapa en el 7,1 \% de sus tuits y con \#1DMX en el 6,9\% de sus tuits.

Algunos de los hashtags asociados con \#YaMeCansé corresponden a marchas y eventos asociados en el que se intenta que sean conocidos y no queden en el anonimato. Tal son las marchas del 20 de noviembre (\#20NovMx) y el 1 de diciembre de 2014: (\#1DMX).

\section{\#YaMeCansé2}

\#YaMeCansé2 así como \#YaMeCansé3, \#YaMeCansé4,..., \#YaMeCansé27 surgen a raíz de que el gobierno federal priista de Enrique Peña Nieto utiliza los llamados bots para censurar hashtags con los que no está de acuerdo. Al censurarlo, una gran cantidad de robots (bots) inundan las redes con tuits sin sentido o que contraponen a lo mencionado, por

Tabla № 4: Popularidad y correlación con \#YaMeCansé2.

\begin{tabular}{lcc}
\hline \multicolumn{1}{c}{ hashtag } & Popularidad & $\begin{array}{c}\text { Correlación } \\
\text { con } \\
\text { \#YaMeCansé2 }\end{array}$ \\
\hline \#YaSuperenlo & 44,7 & $6,4 \%$ \\
\#Ayotzinapa & 62,9 & $5,1 \%$ \\
\#DestitucionyJuicioEPN2 & 44,8 & $5,0 \%$ \\
\#YaMeCansé & 61,4 & $3,7 \%$ \\
\#TodosSomosCompas & 43,5 & $2,8 \%$ \\
\#DestitucionyJuicioEPN & 48,8 & $2,9 \%$ \\
\#6DMx & 40,4 & $2,6 \%$ \\
\#NiUnPesoAlTeleton & 43,5 & $2,4 \%$ \\
\#México & 71,1 & $1,8 \%$ \\
\#TeletonMexico & 41,9 & $1,7 \%$ \\
\#AccionGlobalPorAyotzinapa & 52,6 & $1,5 \%$ \\
\hline
\end{tabular}

ejemplo, en \#YaMeCansé con el objetivo de sacar a ese hashtag de los Trending Topics de Twitter México. En respuesta, en cuestión de minutos los tuiteros mexicanos responden con un nuevo hashtag agregando un número nuevo consecutivo al final de las etiquetas. Sin embargo, así como el original \#YaMeCansé, las subsecuentes etiquetas utilizadas siguen refiriéndose al caso \#Ayotzinapa y al cansancio de los mexicanos por la cantidad de violencia existente en el país. Algunos ejemplos de tuits con \#YaMeCansé2 son:

- \#YaMeCansé2 en México las libertades de las personas están restringidas como en la Alemania Nazi. Llevados a campos de Exterminio.

- Comparecen padres de normalistas ante la ONU \#CasoAyotzinapa \#justicia \#opacidad \#YaMeCansé2

- Parents of murdered Mexican students seek justice at UN watchdog \#Ayotzinapa \#YaMeCanse2 \#Mexico.

Los tres hashtags con mayor popularidad relacionados con \#YaMecansé2 son: 1) \#Mexico con 71,1, 2) \#Ayotzinapa con 62,9 y 3) \#YameCansé con 61,4 de IP.

Al analizar el grado de correlación de \#YaMeCansé2 está asociado con \#YaSuperenlo en el 6,4 \% de sus tuits, con \#Ayotzinapa en el 5,1\% de sus tuits y con \#DestitucionyJuicioEPN2 en el $5 \%$ de sus tuits. En el caso de \#YaMeCansé2 llaman a un bloqueo hacia Televisa, la cadena de televisión más importante en México, debido al apoyo que otorga al actual gobierno. Para ello se usa \#NiUnPesoAlTeleton, \#TeletonMexico.

\section{\#EPNBringThemBack}

El caso de los 43 normalistas desaparecidos se expandió rápidamente por las redes sociales logrando

Tabla № 5: Popularidad y correlación con \#EPNBringThemBack.

\begin{tabular}{lcc}
\hline \multicolumn{1}{c}{ hashtag } & Popularidad & $\begin{array}{c}\text { Correlación } \\
\text { con } \\
\text { \#YaMeCansé2 }\end{array}$ \\
\hline \#regresalosepn & 42,7 & $64,4 \%$ \\
\#sospormexico & 36,3 & $22,7 \%$ \\
\#Ayotzinapa & 62,9 & $12,7 \%$ \\
\#AyotzinapaSomosTodos & 54,4 & $7,8 \%$ \\
\#AccionGlobalAyotzinapa & 43,2 & $5,9 \%$ \\
\#TodosSomosAyotzinapa & 50,8 & $4,8 \%$ \\
\#RegrésalosEPN & 31,8 & $4,4 \%$ \\
\#DemandoTuRenunciaEPN & 46,7 & $4,1 \%$ \\
\#43convidaya & 44,9 & $3,7 \%$ \\
\#regresalospenanieto & 27,7 & $2,5 \%$ \\
\hline
\end{tabular}

la atención de la comunidad internacional. Una de las etiquetas utilizadas llegar al extranjero fue \#EPNBringThemBack. Algunos ejemplos de tuits con \#EPNBringThemBack son:

- \#EPNBringThemBack, el reclamo internacional por caso Ayotzinapa \#Ayotzinapa

- \#MORENA en L.A. se suman a la movilización del \#26DeOctubre \#Ayotzinapa \#EPNbringThemBack \#ConsultaPopular

- \#Ayotzinapa \#EPNBringThemBack: Ultimátum a @ EPN por la desaparición de los 43 estudiantes:

Los tres hashtags con mayor popularidad relacionados con \#EPNBringThemBack son: 1) \#Ayotzinapa que tiene 62,9,2) \#AyotzinapaSomosTodos con 54,4 y 3) \#TodosSomosAyotzinapa con 50,8 de IP. En la tabla 5 se muestra el GC del hashtag \#EPNBringThemBack. 
Este está asociado con \#regresalosepn en el 64,4\% de sus tuits, con \#sospormexico en el $22,7 \%$ de sus tuits y con \#Ayotzinapa en el 12,7\% de los tuits.

\section{\#FueEIEstado}

"Fue el Estado" dice una consigna escrita en el Zócalo de la Ciudad de México. A partir de ahí, esa voz y la protesta se propagaron al mundo entero con el hashtag \#FueElEstado. Este ha aparecido en la gran mayoría de los tuits enviados en el caso Ayotzinapa. Este hashtag retrata una realidad y la visión de una gran mayoría. Algunos ejemplos de tuits con \#FueElEstado son:

- \#Ayotzinapa \#FueElEstado Vivos se los llevaron, vivos los queremos.

- Sabemos lo q hicieron el pasado \#26Sept \#Ayotzinapa \#FueElEstado

Tabla N 6: Popularidad y correlación con \#FueElEstado.

\begin{tabular}{lcc}
\hline hashtag & Popularidad & $\begin{array}{c}\text { Correlación } \\
\text { con } \\
\text { \#FueElEstado }\end{array}$ \\
\hline \#FueraPeñaNieto & 53,1 & $53,3 \%$ \\
\#DestitucionyJuicioEPN & 48,8 & $10,2 \%$ \\
\#AyotzinapaSomosTodos & 54,5 & $9,1 \%$ \\
\#YaMeCanse19 & 47,9 & $7,1 \%$ \\
\#YaMeCanse23 & 44,4 & $6,3 \%$ \\
\#YaMeCanse27 & 43,6 & $6,0 \%$ \\
\#Ayotzinapa & 62,9 & $5,8 \%$ \\
\#YaMeCanse13 & 47,5 & $5,7 \%$ \\
\#YaMeCanse15 & 45,6 & $5,5 \%$ \\
\#YaMeCanse22 & 43,3 & $4,7 \%$ \\
\hline
\end{tabular}

- Lo que reparten los estudiantes en las casetas de cobro \#Ayotzinapa \#RenunciaEPN \#FueElEstado \#43YConVida

- \#FueElEstado Los gobiernos cambian. El Estado siempre es el mismo. \#43VivosYa \#Ayotzinapa

Los tres hashtags con mayor popularidad relacionados con \#FueElEstado son: 1) \#Ayotzinapa, que tiene un IP de 62,9, 2) \#AyotzinapaSomosTodos con 54,5 y 3) \#FueraPeñaNieto con un 53,1 de IP. En la tabla 6 se analiza el GC de \#FueElEstado con los hashtags asociados. En este caso, se puede apreciar que \#FueraPeñaNieto tiene un grado de correlación de $53,3 \%$ con los sus tuits de \#FueElEstado. Por su parte \#DestitucionyJuicioEPN un 48,8 \% y \#AyotzinapaSomosTodos tiene un 9,1 \% de correlación con los tuits de \#FueElEstado.

\section{\#FueElEjercito}

No sólo \#FueElEstado se apoderó de las redes sociales sino también \#FueElEjercito cuando el Procurador General de la República da una versión sobre los hechos que, difícilmente, convence a los padres de los normalistas desaparecidos. Una hipótesis en particular, llama atención: la participación directa del Ejército mexicano en la detención y posterior desaparición de los estudiantes. A partir de esta hipótesis, \#FueEIEjercito crece en popularidad en Twitter. Algunos ejemplos de tuits con \#FueElEjercito son:

- Caso cerrado. Démosle todos \#CarpetazoaEPN. México ya despertó. El futuro es nuestro \#FueEIEjercito

- México en Ginebra: "\#Ayotzinapa es sólo la punta del iceberg" \#fueelestado \#fueelejercito \#fuerapeña \#yamecanse

Tabla N 7: Popularidad y correlación con \#FueElEjercito.

\begin{tabular}{lcc}
\hline \multicolumn{1}{c}{ hashtag } & Popularidad & $\begin{array}{c}\text { Correlación } \\
\text { con } \\
\text { \#FueEIEjercito }\end{array}$ \\
\hline \#FueraPeñaNieto & 53,1 & $68,5 \%$ \\
\#FueElEstado & 55,7 & $29,9 \%$ \\
\#YaMeCanse24 & 42,3 & $27,2 \%$ \\
\#YaMeCanse25 & 43,1 & $23,5 \%$ \\
\#YaMeCanse23 & 44,4 & $18,3 \%$ \\
\#DestitucionyJuicioEPN4 & 46,5 & $18,3 \%$ \\
\#AyotzinapaSomosTodos & 54,5 & $17,8 \%$ \\
\#YaMeCanse3 & 49,9 & $8,1 \%$ \\
\#EselEstado & 45,7 & $6,4 \%$ \\
\#DestitucionyJuicioEPN & 48,8 & $5,4 \%$ \\
\hline
\end{tabular}

- \#AyotzinapaVerdadYJusticia \#FueEIEjercito \#FueElEstado

Los tres hashtags con mayor popularidad relacionados con \#FueElEjercito son: 1) \#FueElEstado, 2) \#AyotzinapaSomosTodos y 3) \#FueraPeñaNieto con $55,7,54,5$ y 53,1 de IP respectivamente.

Analizando la tabla 7 existen algunos hashtags declarativos y en el que hay una consigna precisa como lo es \#FueraPeñaNieto que tiene un grado de correlación del 68,5 \% con los tuits de \#FueElEjercito. También existe una fuerte correlación con \#FueElEstado en el $29,9 \%$ de sus tuits y con \#YaMeCanse24 en el $27,2 \%$ de los tuits.

\section{\#AyotzinapaNoSeOlvida}

Han transcurrido muchos meses desde la desaparición de los estudiantes pero México no los ha olvidado y 
la muestra es el uso de \#AyotzinapaNoSeOlvida que puede encontrarse no solamente en tuits relacionados sino que se usa en diferentes movimientos y en el actual movimiento en México \#EnDefensaDeAristegui. Algunos ejemplos de tuits con \#AyotzinapaNoSeOlvida son:

- \#AyotzinapaNoSeOlvida \#Ayotzinapa de luto

- \#Crónica | Mira aquí la realidad del pueblo de \#Ayotzinapa \#AyotzinapaNoSeOlvida

- \#NoEsPorPresionartePero queremos respuestas @EPN \#Ayotzinapa \#AyotzinapaSomosTodos \#AyotzinapaNoSeOlvida

- \#Ayotzinapa \#AyotzinapaNoSeOlvida El círculo de personas con las que convivo no tiene ni idea del caso \#AyotzinapaFueElEstado

\begin{tabular}{lcc}
$\begin{array}{c}\text { Tabla No 8: Popularidad } \\
\text { \#AyotzinapaNoSeOlvida. }\end{array}$ & $\begin{array}{c}\text { correlación } \\
\text { hashtag }\end{array}$ & con \\
\hline Popularidad & $\begin{array}{c}\text { Correlación } \\
\text { con \#Ayotzinapa } \\
\text { NoSeOlvida }\end{array}$ \\
\hline \#FueElEstado & 55,7 & $31,5 \%$ \\
\#DestitucionyJuicioEPN & 48,8 & $30,7 \%$ \\
\#AyotzinapaSomosTodos & 54,5 & $16,5 \%$ \\
\#Ayotzinapa & 62,9 & $12,3 \%$ \\
\#YaMeCanse & 61,4 & $5,1 \%$ \\
\#YaMeCanse2 & 58,9 & $3,6 \%$ \\
\#TodosSomosAyotzinapa & 50,8 & $3,4 \%$ \\
\#SOSMexico & 44,5 & $3,3 \%$ \\
\#JusticiaParaAyotzinapa & 46,3 & $2,5 \%$ \\
\#México & 71,1 & $2,2 \%$ \\
\hline
\end{tabular}

- Observamos muy detenidamente las acciones del gobierno en el caso \#ayotzinapa \#México \#AyotzinapaSomosTodos \#AyotzinapaNoSeOlvida

Los tres hashtags con mayor popularidad relacionados con \#AyotzinapaNoSeOlvida son: 1) \#Mexico, que tiene un IP de $71,1,2)$ \#Ayotzinapa, que tiene un IP de 62,9 y \#YaMeCanse, que tiene un IP de 61,4.

\#AyotzinapaNoSeOlvida reúne hashtags muy interesantes debido a que hace propuestas, fundamenta las creencias de los ciudadanos y sitúa a la ciudadanía frente a un problema. En esta caso, usa el declarativo \#FueElEstado el cual está correlacionado en un 31,5 $\%$ de los tuits. Se hace una petición mediante los tuits utilizando \#DestitucionyJuicioEPN en el 30,7 \% de los tuits. Por su parte, los ciudadanos no se olvidan de Ayotzinapa y parecido a movimientos anteriores se usa el Yo o el Todos como es \#AyotzinapaSomosTodos aplicado en el 16,5\% de los tuits.
En todos los ejemplos anteriores hay una clara utilización de múltiples hashtags para tratar de contextualizar y ampliar la información ya contenida en el propio tuit. La carencia de un hashtag genérico propicia el uso de otras etiquetas para poder alcanzar un público mayor.

\section{DISCUSIÓN}

Las redes sociales digitales han sido utilizadas como un medio alternativo para pronunciarse en contextos sociales y políticos que antes no permitían involucrar en la discusión a la gran mayoría de la población. Existen números ejemplos en los que, por ejemplo, Twitter ha sido esencial para conocer la actualidad de manos del propio involucrado. En la inmediatez y velocidad generada por las redes sociales es importante analizar el nuevo género narrativo tuit que se caracteriza por la inserción de metadatos en forma de hashtags. Estos permiten darle seguimiento a la gran cantidad de información que se genera minuto a minuto y que son una alternativa para filtrar los tuits pertinentes.

En este artículo se estudió el caso de \#Ayotzinapa surgido en México en septiembre de 2014 y que creció en los meses siguientes debido a su presencia en la red a nivel nacional e internacional. En general, se presenta la evolución del movimiento con los hashtags que fueron surgiendo, en diferentes periodos de tiempo, con el fin de entender cuándo surgen los hashtags y de qué manera permiten seguir manteniendo a la ciudadanía participando en la discusión.

Cada uno de los hashtags analizados muestra la situación actual de México y permiten entender la problemática sin tener que leer cada uno de los tuits generados durante el movimiento.

La evolución de los hashtags y la inserción de varios en un tuit implican varios puntos 1) añadir mayor información al tuit, 2) introducir palabras claves que permiten la recuperación del tuit desde diferentes términos, 3) generar hashtags que sean conocidos por los usuarios para propiciar la generación de comunidad.

El trabajo futuro consiste en el análisis de movimientos sociales similares que puedan conducir a una teoría del hashtag y que permitan detectar y extraer a tiempo cuándo un determinado hashtag puede volverse un Trending Topic. De igual manera, el estudio de hashtags (etiquetas) puede conducir a conocer las características que este debe de tener para poder posicionarse de manera adecuada frente a la gran diversidad de información existente. 


\section{REFERENCIAS BIBLIOGRÁFICAS}

1. Tumasjan A, Sprenger TO, Sandner PG, Welpe IM. Predicting Elections with Twitter: What 140 Characters Reveal about Political Sentiment. International AAAI Conference on Weblogs and Social Media, Washington DC: George Washington University; 2010.

2. Cunha E, Magno G, Comarela G, Almeida V, Gonçalves M A, Benevenuto F. Analyzing the dynamic evolution of hashtags on twitter: a language-based approach. Proceedings of the Workshop on Language in Social Media. Portland: Association for Computational Linguistics; 2011.

3. Romero D, Meeder B, Kleinberg J. Differences in the mechanics of information diffusion across topics: Idioms, political hashtags, and complex contagion on Twitter. 20th International World Wide Web Conference. Hyderabad: ACM; 2011.

4. Chew C, Eysenbach G. Pandemics in the age of Twitter: Content analysis of tweets during the 2009 H1N1 outbreak. PLoS ONE. 2010; 5(11): 1-13.

5. Barbosa GAR, Silva IS, Zaki MJ, Meira Jr. W, Prates RO, Veloso A. Characterizing the effectiveness of twitter hashtags to detect and track online population sentiment. Proceedings of the 2012 ACM annual conference extended abstracts on Human Factors in Computing Systems Extended Abstracts. Austin: ACM; 2012.

6. Zappavigna M. Ambient affiliation: A linguistic perspective on Twitter. New media \& society, 2011; 13(5): 788-806.
7. Bruns A, Burgess JE. The use of Twitter hashtags in the formation of ad hoc publics. 6th European Consortium for Political Research General Conference. Reykjavik: University of Iceland; 2011.

8. FactoryCity. 2007 Aug 25 [cited 2015 July 10]. In: Twitter Hashtags for Emergency Coordination and Disaster Relief [Internet]. Available from: http://factoryjoe.com/blog/2007/10/22/twitterhashtags-for-emergency-coordination-anddisaster-relief/

9. Hyland K, Tse P. Metadiscourse in academic writing. Applied Linguistics. 2004; 25(2): 156-177.

10. Crismore A, Markannen R, Steffensen $M$. Metadiscourse in persuasive writing: A study of texts written by American and Finnish university students. Writ Commun. 1993; 10(1): 39-71.

11. Russell A. The Arab Spring Extra-National Information Flows, Social Media and the 2011 Egyptian Uprising. International Journal of Communication. 2011; 5: 1238-1247.

12. Poell T, De Kloet J, Zeng G. Will the real Weibo please stand up? Chinese online contention and actor-network theory. Chinese Journal of Communication. 2014; 7(1): 1-18.

13. Gitlin T. The whole world is watching: mass media in the making \& unmaking of the new left. London: University of California Press; 2003.

14. Georgakopoulou A. Narrative analysis and computer-mediated communication. In: Herring S, Stein D, Virtanen T. Pragmatics of ComputerMediated Communication. Berlín: Illustrated; 2013. 